\section{Anisotropy of Diffusion in Rutile}

DuRING the determination of diffusion parameters in the system $\mathrm{Fe}_{2} \mathrm{O}_{3}-\mathrm{TiO}_{2}$, marked anisotropy was found in the diffusion of $\mathrm{Fe}$ (III) into a rutile single erystal. Single crystals of rutile and haematite were cut and polished to one micron on successive diamond laps. The polishing treatment did not result in perfectly plane surfaces but in convex surfaces which gave contact between the crystals over only a relatively small area when they were brought into contact to form diffusion couples (Fig. 1). Annealing was carried out in a flowing oxygen atmosphere at: $1,100^{\circ} \mathrm{C}$ for $20 \mathrm{~h}$.

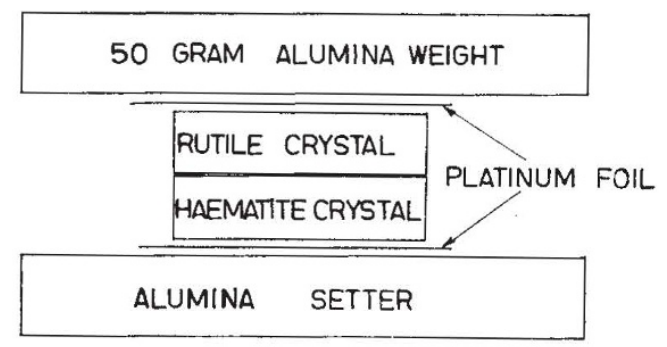

Fig. 1. Diffusion couple assembly.
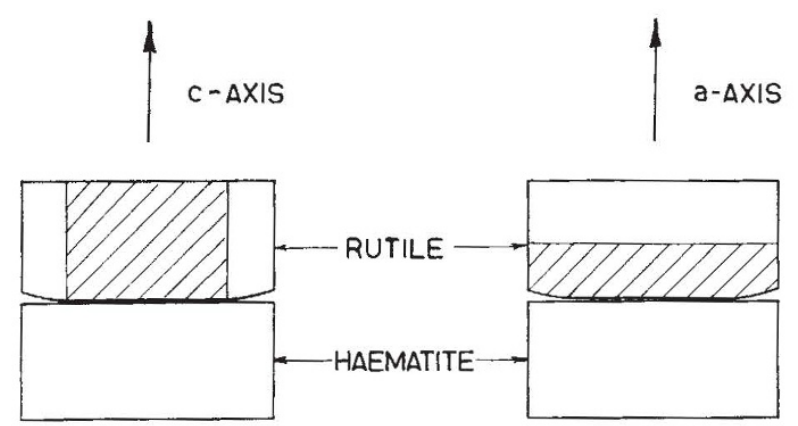
Fig. 2. Diffusion front characteristics in a and c-axis rutile crystals
Shaded area is diffused region.

The couples were then sectioned parallel to the direction of diffusion and polished for electron microprobe analysis. This showed that the diffusion front had a characteristic form dependent on the orientation of the rutile crystal in relation to the haematite crystal. The effect is shown schematically in Fig. 2 in which the edge curvature has been exaggerated for clarity. The presence of concentrations of iron in the rutile as low as 0.4 per cent produces a deep red colour so that the diffusion front shows up as a very sharp colour boundary. When the $c$-axis of the rutile crystal is normal to the interface, the diffusion is confined to a sharply defined region of the same cross-section as the contact area. There is very little "fanning out" of the iron; diffusion seems to be unidimensional. When the a-axis of the rutile is normal to the interface, however, diffusion still occurs preferentially in the c-direction. Diffusion in the a-direction seemed to be negligible until the layer parallel to the interface had been saturated. All the evidence thus indicates a much higher rate of diffusion along the c-axis than along the a-axis.

There are reports of similar behaviour during the diffusion of other ions. Lithium was found ${ }^{1}$ to diffuse along the c-axis of a rutile crystal by a factor of up to $10^{8}$ times faster than along the a-axis. A higher rate of diffusion along the c-axis was also reported for $\mathrm{Ba}$ (ref. 2) although the magnitude of the difference was not reported. In contrast, diffusion of $\mathrm{O}$ was found ${ }^{3}$ to be faster along the a-axis than the c-axis. It would seem that this behaviour is related to the crystal structure. Rutile has channels of low electron densities lying along the centres of squares made up of $\mathrm{Ti}$ ions along the $\mathrm{c}$-axis and it might be expected that cations could move more easily along this "open" direction than along the more restricted a-direction. These considerations apply strictly to interstitial diffusion, but there is some evidence ${ }^{4}$ to suggest that this is in fact the diffusion mechanism for transition metal ions in rutile.

\section{J. L. SteEle \\ E. R. McCartiney}

Department of Ceramic Engineering,

University of New South Wales,

Kensington, New South Wales.

Received February 10, 1969.

1 Johnson, O. W., Phys. Rev., 136, 284 (1964).

2 Nakayama, T., and Sasaki, T., Bull. Chem. Soc. Japan, 36, 569 (1963).

${ }^{3}$ Haul, R., and Duembgen, G., J. Phys. Chem. Solids, 26, 1 (1965).

${ }^{4}$ Wittke, J. P., J. Electrochem. Soc., 113, 193 (1966).

\section{Growth of Single Crystals of Cuprous Oxide in Silica Gels at Near Ambient Temperatures}

Crystal growth in gels is becoming increasingly rocognized as a promising technique for the preparation of single crystals of substances which are slightly soluble in water and which cannot be conveniently grown from the melt or from the vapour ${ }^{1}$. The growth of single erystals of many metal oxides is of considerable interest, particularly for basic studies of their electronic properties and other pertinent characteristics. Single crystal growth in gels at near ambient temperatures offers a possibility of obtaining specimens free from thermal strains and the impurities frequently associated with high temperature preparative methods. The preparation of single crystals of metal oxides in gels at low temperatures has not previously been successfully achieved, probably because of a strong tendency of metal oxides to form hydrated polycrystalline or amorphous precipitates in such media. We report the growth of single crystals of cuprous oxide in silica gels at near ambient temperatures by the controlled reduction of cupric sulphate with selected mild reducing agents such as hydroxylamine hydrochloride and hypophosphorous acid.

Silica gels for use in growing single crystals of cuprous oxide were prepared by mixing equal volumes of sodium silicate solution of specific gravity 1.06 (Fisher certified reagent) and acetic acid $(1 \cdot 6 \mathrm{~N})$. Approximately $1 \mathrm{ml}$. of a $1 \mathrm{~N}$ solution of cupric sulphate was added for each $10 \mathrm{ml}$. of this mixture. The resulting $p \mathrm{H}$ ranged from $4 \cdot 7-4 \cdot 8$. This solution was poured into standard $25 \mathrm{~mm}$ diameter test tubes and allowed to gel at $35^{\circ} \mathrm{C}$-a process which required 24-36 h. An additional amount of the gel forming mixture, similarly made by mixing equal volumes of 1.06 specific gravity sodium silicate solution and $1.6 \mathrm{~N}$ acetic acid but containing no cupric sulphate, was placed on this gel and in turn allowed to gel at $35^{\circ} \mathrm{C}$. The ratio of the original gel volume to this second top gel volume was approximately $1: \mathrm{I}$.

The resulting two layer gel system was covered with a 1 per eent solution of a reducing agent such as hydroxylamine hydrochloride or hypophosphorous acid. The amounts of reducing agent solutions used to cover the gels were approximately $10 \mathrm{ml}$. on $75 \mathrm{ml}$. of total gel. Cubic crystals of cuprous oxide grew slowly with equilibrium established after about 2 weeks, the size of the crystals being about $0.5 \mathrm{~mm}$ on edge. When crystals were kept in the gels for 4-6 weeks, copper films began to form on the surface of the cuprous oxide crystals.

The use of hypophosphorous acid as the reducing agent gave better uniformity and generally larger crystal sizo than did hydroxylamine hydrochloride. Using this pro- 